\title{
Research and Application of Image Recognition of Substation Inspection Robots based on Edge Computing and Incremental Learning
}

Xiao Liu ( $\nabla$ xia00856159@163.com )

Maintenance Branch of State Grid Shanxi Electric Power Company

Bangzhou Dong

Maintenance Branch of State Grid Shanxi Electric Power Company

Peiqi Li

Maintenance Branch of State Grid Shanxi Electric Power Company

Bin Yuan

Maintenance Branch of State Grid Shanxi Electric Power Company

Kesheng Wang

maintenance Branch of State Grid Shanxi Electric Power Company

\section{Research Article}

Keywords: edge computing, incremental learning, inspection robot, image recognition

Posted Date: February 8th, 2021

DOI: https://doi.org/10.21203/rs.3.rs-172739/v1

License: (9) This work is licensed under a Creative Commons Attribution 4.0 International License.

Read Full License 


\title{
Research and application of image recognition of substation inspection robots
} based on edge computing and incremental learning

\author{
Xiao Liu*, Bangzhou Dong, Peiqi Li, Bin Yuan, Kesheng Wang
}

Maintenance Branch of State Grid Shanxi Electric Power Company, Taiyuan, Shanxi 030006,

China

Corresponding address: No. 9, Jiahua Street, Xiaodian District, Taiyuan, Shanxi 030006, China

Email: xia00856159@163.com 


\title{
Research and application of image recognition of substation inspection robots based on edge computing and incremental learning
}

\begin{abstract}
:
The large scale of the power grid and its high voltage make the fault detection have not only heavy workload but also high risks. This paper briefly introduced the inspection robot system, edge computing technology, and support vector machine (SVM)-based image recognition algorithm. To enable the inspection robot system to actively adjust the algorithm parameters and maintain the accuracy of the algorithm, the SVM algorithm was improved by the incremental learning technology. Then, the nonimproved and improved SVM algorithms were simulated in MATLAB software. A one-month experiment was carried out in a substation. The results showed that the improved SVM algorithm trained faster and had a high accuracy in the face of new samples. The substation experiment verified that the inspection robot system maintained the infrared image recognition accuracy of the faulty equipment for a long time after the application of the improved SVM algorithm, and the system had a smaller delay in transmitting command information after the application of edge computing.
\end{abstract}

Keywords: edge computing, incremental learning, inspection robot, image recognition

\section{Introduction}

With the rapid development of society, more and more energy is needed in the process of development. As a stable and relatively clean energy, electricity has been widely used in daily life and production. With the expansion of energy demand, the transmission capacity of the power grid also increases, and the scale of supporting substations is becoming larger and larger. The increase in the size of substations leads to the corresponding increase in the number of power equipment, which not only improves the transmission capacity of the power grid but also increases the risk of equipment failure [1]. For the substation, due to the large amount of power energy transported, once the failure occurs, a large area of power will be cut off, or even fire will be caused, resulting in heavy loss of people's lives and property [2]. Therefore, the daily maintenance of substation power facilities is very important. The traditional maintenance relies on human patrol. In the face of the gradually increasing size of the substation, the cost of human management also rises. The appearance of the inspection robot greatly liberates the labor force, and its intelligent recognition algorithm can detect equipment faults more objectively and efficiently. Zhao et al. [3] proposed a combined orbital intelligent inspection robot, then combined with the linear programming algorithm to solve the problem, and finally applied it in the water cooling room of a substation. The results showed that the robot could meet the requirements of on-site inspection. $\mathrm{Xu}$ et al. [4] proposed an obstacle avoidance method based on deep learning and depth image for the substation inspection robot and verified the effectiveness of the method by the actual test in the actual substation environment. Jiang et al. [5] designed an intelligent detection robot according to the 
background of substation indoor equipment and the requirements of detection content, carried out practical application tests, and found that the orbital inspection robot had a reasonable structure and reliable operation. This article briefly introduces the inspection robot system, edge computing, and support vector machine (SVM)-based image recognition algorithm. The SVM algorithm was improved by the incremental learning technology, in order to make the inspection robot system be able to actively adjust the algorithm parameters and maintain the accuracy of the algorithm. The original and improved SVM algorithms were simulated in MATLAB software. A one-month experiment was carried out in a substation.

\section{Inspection robot and edge computing}

In the face of the increasing scale of substations, the human cost of fault inspection is also increasing. The recognition rate of human inspection for substation equipment fault mainly depends on the work experience of inspectors. Secondly, the record of substation equipment fault also needs a large number of paper record sheets, which is not only troublesome in recording but also a great waste of paper. With the development of wireless communication technology and intelligent algorithms, inspection robots have been gradually applied in substation equipment fault inspection. The system framework of inspection robots can be roughly divided into the cloud service layer that provides services, such as computing, planning, etc., the network communication layer that is used for transmitting information and data, and the terminal layer (inspection robot) that is used for collecting fault information of substation equipment. The inspection robot (the terminal layer), which carries out inspection works in the substation, collects fault information of substation equipment with its equipment, such as infrared camera, ordinary camera, radio frequency identification (RFID) sensor, etc. [6]. Then, the information is uploaded to the cloud device and the main power control system in the cloud service layer through the wireless network of the network communication layer. After the judgment by the relevant systems in the cloud service layer, the corresponding instructions are sent back to the terminal layer. The above process is a flow of a relatively conventional inspection robot system. The use of wireless network realizes the free inspection; however, the effective transmission range of the wireless signal is limited, cloud computing resources mainly concentrate on the network center, and the assignable computing resources in the peripheral edge are limited. Thus, the information collected by the robot still needs to be transmitted to the network center server through multi-hop nodes for processing, which causes delay. In general scenarios, the delay does not have much impact, but timely fault detection and feedback in the substation is the key to ensure safety; therefore, the transmission and processing of fault information need to be as fast as possible. 


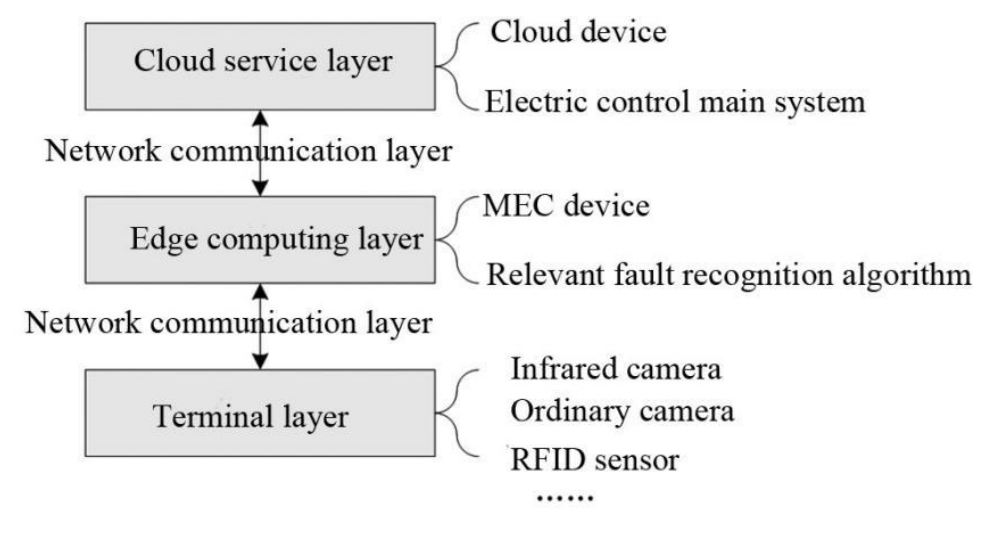

Figure 1 The system framework of the substation inspection robot based on edge computing

The wireless network used by the inspection robot system has limited coverage. The farther away the inspection robot is from the network service center, the fewer resources it can allocate, and the larger the delay is [7]. After the complex robot collects fault information and transmits it to the central server, the burden of the resource-limited server will increase, which will eventually affect the accuracy of fault judgment. In order to reduce the burden of the central server and the delay, edge computing technology is introduced into the original inspection robot system. Edge computing technology refers to setting an open platform with the same functions, such as network communication, computing, and storage, at the network edge near the network center to share a part of data processing tasks. Compared with the traditional cloud computing model, the edge computing model has better bearing capacity for big data and can achieve multi-task parallel processing by combining with the central device, which can effectively improve processing efficiency [8]. Figure 1 shows the framework of the substation inspection robot system after adding edge computing technology. Compared with the traditional framework, the edge computing layer is added, and the rest of the structure has not changed too much. The cloud service layer is still the system center providing computing services, including cloud devices and control systems. The terminal layer is still the inspection robot, including a variety of information collection devices. The added edge computing layer is the edge network in the whole network system, usually composed of small base stations, i.e., mobile edge computing (MEC) devices. These base stations not only undertake the function of information transmission but also has functions of computing and storage; moreover, they also have relevant recognition algorithms for substation failures.

The basic process of the edge computing-based substation inspection robot system for the intelligent inspection of a substation is as follows. (1)The staff log in the power control system in the cloud service layer and assign inspection tasks to the inspection robot in the terminal layer. (2)After the command of inspection task is transferred to the edge computing layer, MEC devices in this layer will detect the number of nearby robots and assign corresponding inspection tasks. 3The inspection robot start to detect the equipment after receiving the instruction, mostly by infrared thermal imaging; the robot uses the RFID sensor to determine the location and number of the detected equipment and collect the infrared image of the equipment by the infrared camera. (4)The terminal layer will transmit the collected infrared images to the MEC device of the edge computing layer after simple preprocessing, the images are 
identified using the corresponding fault image recognition algorithm, and the judgment results are transmitted to the cloud device of the cloud service layer; the MEC device will direct the terminal layer to make a preliminary warning according to the recognition results; more specific operations are determined by the cloud service layer according to the recognition results uploaded by MEC devices.

3. The incremental learning-based SVM algorithm for image recognition

3.1 SVM algorithm for image recognition

The edge computing-based inspection robot system has been briefly introduced above. Compared with the traditional inspection robot system, the system added with edge computing technology no longer identifies and processes the fault images in the central server when facing the infrared images collected by the robot. Instead, the image recognition task is assigned to the MEC server closer to inspection robots at the network edge, which reduces the workload of the central server, improves the volume of data processing, and enhances the timeliness.

For the substation inspection robot, whether it is the traditional system or the system added with edge computing technology, the core is recognizing infrared images of substation equipment, and this function needs to be achieved by corresponding fault recognition algorithms. Although the infrared images collected by the inspection robot can be judged manually, it is not practical in the application of substation inspection. On the one hand, the efficiency of manual recognition is low because of the large number of images; on the other hand, the accuracy of manual recognition depends on experience, leading to poor stability. The improvement of computer performance provides a good foundation for the realization of intelligent recognition algorithms. The SVM algorithm is a common intelligent classification algorithm [9], which can be used for identifying the fault of equipment in infrared images. The principle of the SVM algorithm for classification and recognition of samples is to find the "hyperplane" in feature vector space and divide the feature vector space into different regions. Each region is a classification.

In this study, the SVM algorithm can not recognize and classify the infrared images collected by inspection robots directly. Firstly, it needs to extract the image features. In this study, scale-invariant feature transform (SIFT) features are used as image recognition and classification features, the feature scale trajectory curve is obtained by the DoG operator, and the extreme points are obtained from the curve. The calculation formula of the curve [10] is:

$$
D(x, y, \sigma)=(G(x, y, k \sigma)-G(x, y, \sigma)) \bullet I(x, y)=L(x, y, k \sigma)-L(x, y, \sigma),
$$

where $G(\bullet)$ is a Gaussian function, ${ }^{k}$ is the gain constant, ${ }^{I(x, y)}$ is the input image, ${ }^{x, y}$ is the coordinate set of image pixels, and $L(\bullet)$ is the scale space. Then, the histogram of the neighborhood gradient direction of all extreme points is counted, and the SIFT feature vector of the recognition image is obtained after combining the histograms. Then, the features of the image were integrated into training 
samples. The SVM algorithm calculates the decision function using training samples in the process of training, and the calculation formula is:

$$
\left\{\begin{array}{l}
f(x)=\operatorname{sgn}\left(\sum_{i=1}^{l} a_{i} y_{i} K\left(x_{i}, x_{j}\right)+b\right) \\
\sum_{i=1}^{l} a_{i} y_{i}=0 \quad 0 \leq a_{i} \leq C
\end{array}\right.
$$

where $a$ is the set of ${ }^{a}, a_{i}$ is the Lagrange coefficient [11], $l$ is the sample size, $K\left(x_{i}, x_{j}\right)$ is a kernel function, $C$ is the penalty parameter, ${ }^{y_{i}}$ is the result of classification, and $x_{i}$ is the sample data.

\subsection{Improvement of SVM algorithm by incremental learning}

Compared with other intelligent recognition algorithms, the SVM algorithm can map low-dimensional sample features to the high-dimensional vector space through the kernel function, thus transforming a nonlinear solving problem into a linear programming problem, which avoids locally optimal solution and has a fast convergence during training. The SVM algorithm has the above advantages in intelligent classification recognition; however, in the actual application, the influence of the outer world on infrared image characteristics of substation faults is not a definite value but a floating range. The SVM training should ensure not only the accuracy but also the training efficiency; therefore, the size of the training sample needs to compromise between them. But in any case, the parameters of the SVM algorithm obtained by the limited sample training can not entirely fit in with realistic classification standards, and new samples should be constantly added to update the parameters, making it keep accurate classification criteria as far as possible. However, the SVM algorithm does not support incremental learning. In the face of new samples, it needs to retrain the new samples after integration with the original samples, which eventually leads to a large sample set, increases training time, and improves storage requirements.

In order to solve this problem, the SVM algorithm was improved by incremental learning. When the improved SVM algorithm faces new samples, it does not need to train the existing training samples repeatedly but gradually accumulates new samples to maintain or improve the recognition accuracy. 


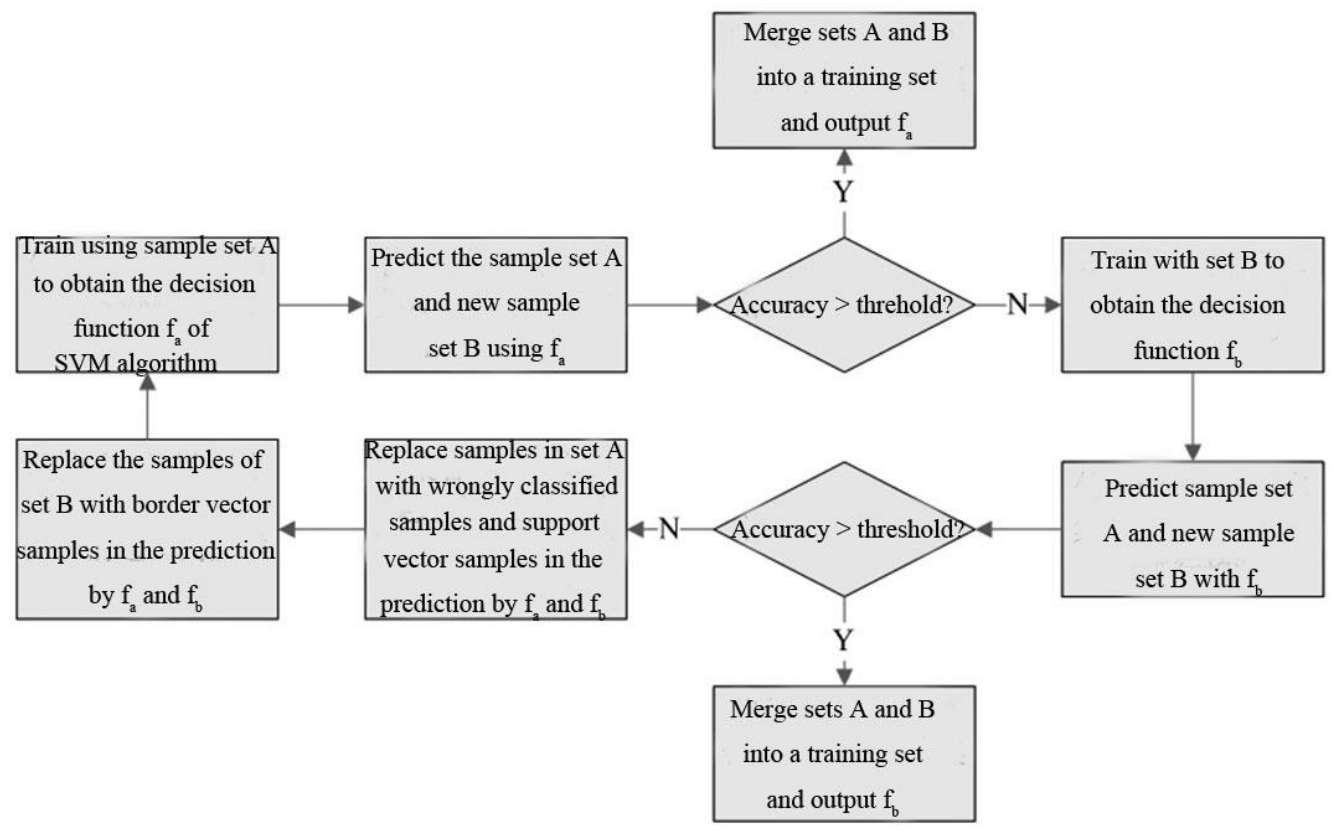

Figure 2 The training process of the improved SVM algorithm

The training process of the improved SVM algorithm is shown in Figure 2. In the face of the new sample set, the improved SVM algorithm no longer integrates it into the original sample set for retraining but trains it with the incremental learning method. Then, it makes a choice between the original decision function and the new decision function according to the KTT condition [12] and border vector so that the final decision function can accurately divide the original sample and new sample. The specific flow is as follows.

(1)After SIFT features are extracted from the training sample set A, the decision function $f_{a}$ is obtained according to the conventional SVM training method.

(2)The classification of set $\mathrm{A}$ and new sample set $\mathrm{B}$ are predicted using $f_{a}$, and the overall recognition accuracy of sets A and B by $f_{a}$ is recorded.

(3) If the accuracy of $f_{a}$ exceeds the threshold, then set $\mathrm{A}$ and $\mathrm{B}$ are merged, and $f_{a}$ is taken as the decision function of the SVM algorithm after incremental learning; if it does not, then only the new set B is used for training to obtain the decision function $f_{b}$ of the SVM algorithm.

(4)The classification of set $\mathrm{A}$ and new sample set $\mathrm{B}$ are predicted using $f_{a}$, and the overall recognition accuracy of sets $\mathrm{A}$ and $\mathrm{B}$ by $f_{b}$ is recorded. 
(5)If the accuracy of $f_{b}$ exceeds the threshold, then set $\mathrm{A}$ and $\mathrm{B}$ are merged, and $f_{b}$ is taken as the decision function of the SVM algorithm after incremental learning; if it does not, then the samples of set A are replaced by the wrongly classified samples and support vector samples in the prediction made by $f_{a}$ and $f_{b}$. The samples of set $\mathrm{B}$ are replaced by the border vector samples in the prediction made by $f_{a}$ and $f_{b}$. Then, the replaced samples A and B return to step (1) for cycling.

In the process described above, if the accuracy of $f_{a}$ or $f_{b}$ can exceed the threshold, the final decision function can be easily obtained, but if both can not exceed the threshold, sets A and B need to be adjusted to recalculate decision functions $f_{a}$ and $f_{b}$. The wrongly classified samples used for replacing set A refer to the samples that are wrongly classified when sets $\mathrm{A}$ and $\mathrm{B}$ are classified by $f_{a}$ and $f_{b}$. Supporting vector samples are on decision functions $f_{a}$ and $f_{b}$, which can be simply regarded as decision functions. The boundary vector samples used for replacing set B are those which satisfy the KTT condition, are closer to the decision function, and may become part of the decision function in the subsequent cycle training.

\section{Experimental analysis}

\subsection{Simulation environment}

In this study, the improved and non-improved SVM image recognition algorithms in the inspection robot were simulated in the MATLAB software [13]. Then, a one-month experiment was carried out in No. 1 substation of X City Power Supply Bureau to test the improvement of image recognition performance of the inspection robot system after adding edge computing technology. The simulation experiment was carried out in a laboratory server with configurations of Windows 7 system, I7 processor, and $16 \mathrm{G}$ memory.

\subsection{Experimental data}

In this study, the infrared image data used in the simulation experiment was provided by the power supply bureau of X city. There were 10000 infrared images, including five types of power equipment; 5000 infrared images contained abnormal hot spots, i.e., equipment failure images. After the infrared images are rotated, zoomed, and flipped, many new images were obtained. $70 \%$ of the images were used as the training set, and the remaining $30 \%$ were used as the testing set. Through the orthogonal test in advance, the radial basis function was selected as the kernel function $K\left(x_{i}, x_{j}\right)$ of the SVM algorithm [14]: 
$k\left(x_{i}, x\right)=\exp \left(-\frac{\left\|x-x_{i}\right\|^{2}}{\sigma^{2}}\right)$

The penalty factor $C$ [15] was set as 8 .

In the substation experiment, the basic parameters of the inspection robot used for collecting the infrared images of substation equipment are as follows. The height of the main body height was $1.5 \mathrm{~m}$, the size of the chassis was $1 m \times 0.7 m$, the overall weight was $80 \mathrm{~kg}$, the movement accuracy was within $2.0 \mathrm{~cm}$, and the maximum transmission range of wireless signal was $10 \mathrm{~km}$. The top camera had an infrared temperature measurement function. The robot chassis was installed with RFID sensors for sensing and identifying its location. The robot moved in the driving wheel and adjusted the direction through the follower wheel. In addition, a wireless communication base station was set every $20 \mathrm{~m}$ in the inspection area of the inspection robot in the substation. In addition to the communication function, these base stations also have the ability to calculate and store. The base stations were equipped with Inter E5 CPU, NVIDA 1080 Ti GPU, 128 G random access memory, and Windows 7.

\subsection{Experimental project}

\section{(1) Simulation experiment}

In this study, before the improved SVM image recognition algorithm was applied to the inspection robot, a simulation experiment was carried out in a laboratory server. In the process of the simulation experiment, the images in the training set and the testing set have been preprocessed, and SIFT features have been extracted. To verify the improvement of infrared image recognition performance of the SVM algorithm improved by incremental learning technology, the simulation experiment also tested the traditional SVM algorithm besides the improved SVM algorithm. The kernel function and penalty factor of the traditional SVM algorithm were consistent with those of the improved SVM algorithm. The training set was randomly divided into five parts, one of which was the original training set, and the remaining four parts were incremental samples. The improved and non-improved SVM algorithms were trained by the original training set and tested by the testing set; the accuracy and training time were recorded. Then, the incremental samples were added to the original training set four times for the incremental training of the improved SVM algorithm, and it was tested by the testing set after every time of training. The traditional SVM algorithm also added the incremental samples into the original training set four times, but was trained by the training set rather than by the incremental training; it was tested by the testing test after every time of training.

\section{(2) Substation experiment}

In the substation experiment, the improved and non-improved SVM image recognition algorithms were tested, and the improvement of the performance of the inspection robot image recognition system by edge computing technology was also tested. The common experimental steps are as follows. The 
inspection robots were put on the designated initial positions, and the moving route was set as a route that could detect all the power equipment in the substation. The area where the power equipment was located was divided, and every area was configured with a corresponding RFID tag. In the inspection process, the inspection robot identifies its location through the RFID sensor. When the electronic tag of the power equipment was scanned, the robot stopped and used the camera to take pictures of the power equipment.

The following experiment steps were different according to the setting of experiment items. The experiment in the substation needed to test not only the actual performance of the non-improved and improved SVM algorithms for image recognition but also the influence of edge computing technology on the whole inspection robot image recognition system; therefore, the experimental items for comparison were set as follows. (1)The infrared images collected by the robots were transmitted to the master server in the control room through the base station, and the master server used the traditional SVM algorithm to recognize the images and transmitted the alarm signal to the robot according to the result; (2)the infrared images were transmitted to the master server through the base station, and the master server used the improved SVM algorithm to recognize the image and transmitted the alarm signal to the robot according to the result; (3)the infrared images were identified by the traditional SVM algorithm in the base station, the recognition results were uploaded to the master server, and the alarm signal was sent to the robot according to the result; (4 the infrared images were identified by the improved SVM algorithm in the base station, the recognition results were uploaded to the master server, and the alarm signal was sent to the robot according to the result.

The substation maintenance personnel used the hand-held infrared detection equipment to confirm the identification results and carried out regular substation maintenance and detection. The maintenance personnel who participated in this experiment had more than five years of working experience.

\subsection{Experimental results}

Although the improved SVM algorithm adopted the incremental learning technology, its structure and training process differed little from the traditional SVM algorithm in the face of a single training sample. Therefore, the comparison of the infrared image recognition performance between the two algorithms after training should be carried out after multiple incremental training. Figure 3 shows one of the recognition results of the two SVM algorithms after four times of incremental training. It was seen from Figure 3 that the substation equipment had two abnormal hot spots and the abnormal hot spot in the middle had a higher temperature and was more obvious. The traditional SVM algorithm only identified the more obvious hot spot, but failed to recognize the other hot spot. The improved SVM algorithm not only identified the more obvious abnormal hot spot but also identified the relatively less obvious hot spot. 


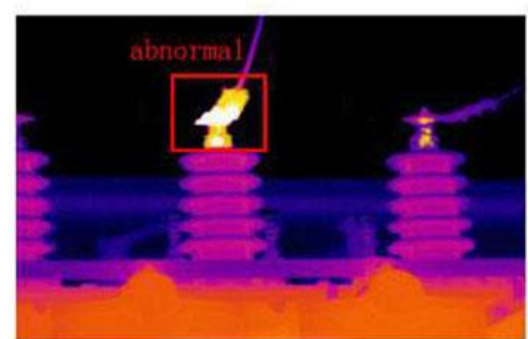

Traditional SVM algorithm

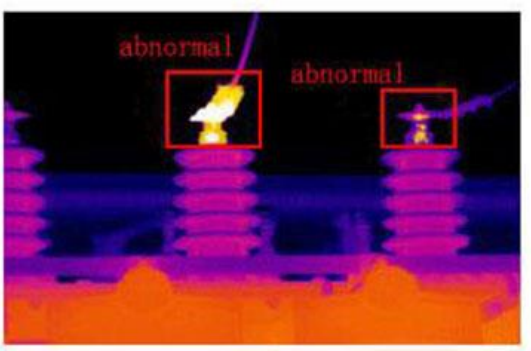

Improved SVM algorithm

Figure 3 Recognition results of two SVM algorithms for infrared images after incremental learning

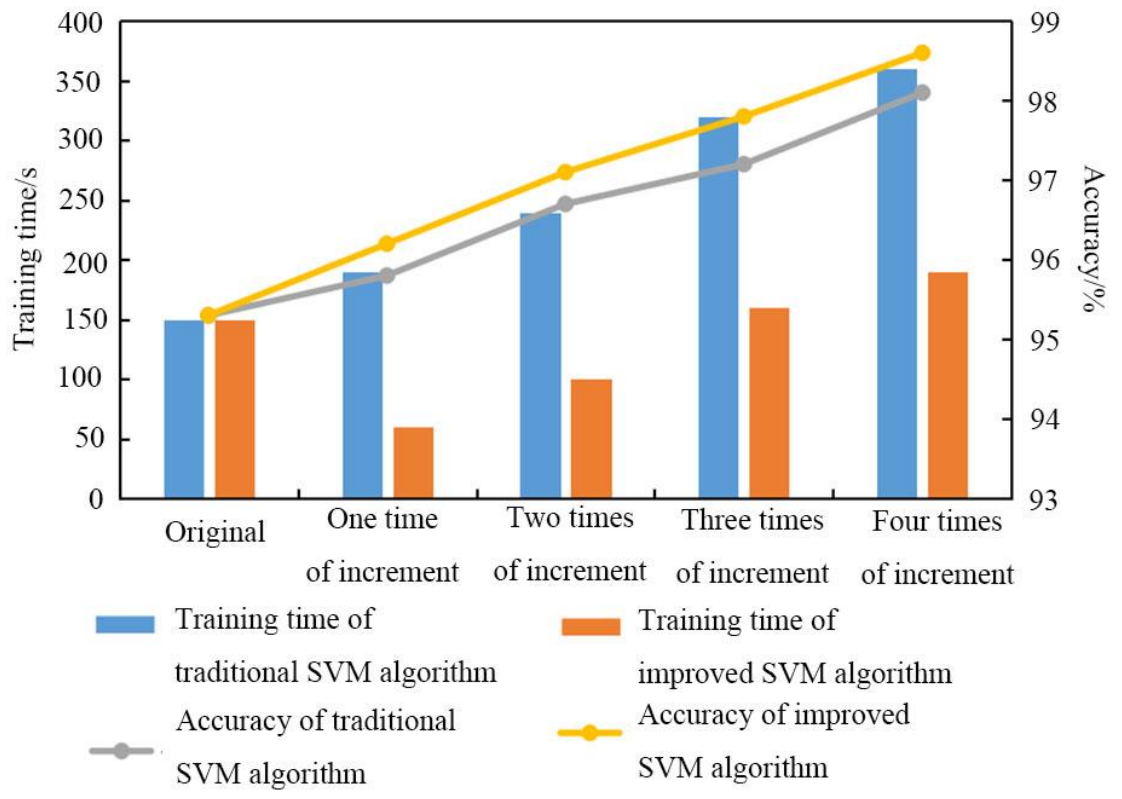

Figure 4 Results of the simulation experiment

The simulation results in the laboratory are shown in Figure 4. It was seen from Figure 4 that the training time and accuracy of the two SVM algorithms were the same under the original training set, which was because the two SVM algorithms had the same training method, parameters, and samples. After the incremental training, the training time of the traditional SVM algorithm increased with the increase of incremental samples, and the training time of the improved SVM algorithm decreased first and then increased, but was significantly shorter than that of the traditional SVM algorithm. The reason for the above result was that the traditional SVM algorithm added the incremental samples into the original samples and trained them again, and the larger the increment was, the larger the size of the training sample was; however, the improved SVM algorithm gradually adjusted the decision function according to the incremental samples and did not train the original training samples. The increase of the training time of the improved SVM algorithm was because that the original and incremental samples were 
predicted when the decision function was adjusted, and the increase of the prediction amount resulted in the increase of the training time.

The accuracy of both traditional and improved SVM algorithms improved with the increase of incremental samples after training. The reason was that more samples could reflect more accurate rules. In the aspect of numerical values, the accuracy of traditional and improved SVM algorithms was above 95\%, and the improved SVM algorithm was slightly superior.

Table 1 Influence of edge computing technology and incremental learning technology on image recognition of inspection robots in substation experiment

\begin{tabular}{|c|c|c|c|c|}
\hline & $\begin{array}{l}\text { Actual failure } \\
\text { times/time }\end{array}$ & $\begin{array}{l}\text { Accurate } \\
\text { times/time }\end{array}$ & $\begin{array}{l}\text { Number of false } \\
\text { positives/n }\end{array}$ & $\begin{array}{l}\text { Average delay of } \\
\text { alarm } \\
\text { instruction/ms }\end{array}$ \\
\hline $\begin{array}{l}\text { Traditional SVM } \\
\begin{array}{l}\text { algorithm without } \\
\text { edge computing }\end{array}\end{array}$ & 87 & 74 & 16 & 85 \\
\hline $\begin{array}{l}\text { Improved SVM } \\
\text { algorithm without } \\
\text { edge computing }\end{array}$ & & 85 & 4 & 84 \\
\hline $\begin{array}{l}\text { Traditional SVM } \\
\text { algorithm with edge } \\
\text { computing }\end{array}$ & & 75 & 14 & 21 \\
\hline $\begin{array}{l}\text { Improved SVM } \\
\text { algorithm with edge } \\
\text { computing }\end{array}$ & & 86 & 2 & 20 \\
\hline
\end{tabular}

The results of the actual experiment in the substation are shown in Table 1. Through manual inspection, 87 abnormal heating faults of the substation equipment were found in one month. It was seen from the comparison in Table 1 that the accurate times were more and closer to the actual failure times and the number of false positives was less when the improved SVM algorithm was used for recognizing the infrared images no matter edge computing technology was used or not. The reason was that the faults generated by substation equipment were completely consistent with those in the samples in the actual application process, and new fault features generated; the traditional SVM algorithm that did not support incremental learning gradually deviated from the accurate standard in the process of the long-term use, but the improved SVM algorithm that supported incremental learning added the fault images collected in the operation process as the samples and constantly adjust the decision function to make it close to the accurate standard.

Under the same SVM image recognition algorithm, whether edge computing technology was used or not, the accurate recognition times and the number of false positives of the two algorithms were very close, 
but the average delay of alarm instruction was smaller in the inspection robot system that adopted edge computing technology. The reason why the accuracy was close under the same algorithm was that the recognition of infrared images depended more on the image recognition algorithm in the system. After using the edge computing technology, the system assigned recognition tasks to the edge base stations for parallel processing, which improved the recognition efficiency; the nodes that needed to hop were less as the edge base stations were closer to the inspection robots, which made the delay of instruction signals smaller.

\section{Conclusion}

This paper briefly introduced the inspection robot system, edge computing technology, and SVM image recognition algorithm. Then, to enable the inspection robot system to actively adjust the algorithm parameters and maintain the accuracy of the algorithm in the process of using, the SVM algorithm was improved by the incremental learning technology. Then, original and improved SVM algorithms were simulated in MATLAB software, and a one-month experiment was carried out in a substation. The results are as follows. In the simulation experiment, with the increase of incremental samples, the training time and recognition accuracy of the traditional SVM algorithm increased, the training time of the improved SVM algorithm first decreased and then increased, but was shorter than the traditional SVM algorithm, and the accuracy of the improved SVM algorithm increased, slightly better than the traditional SVM algorithm. In the substation experiment, whether edge computing was used or not, the fault recognition times of the inspection robots based on the improved SVM algorithm was closer to the actual fault times, and the number of false positives was also smaller; under the same SVM algorithm, although the inspection robot system that adopted edge computing had little difference in fault recognition accuracy, the average delay of alarm instructions was smaller.

\section{Declarations}

Availability of data and materials: The data that support the findings of this study are available from the corresponding author upon reasonable request.

Competing interests: No conflict of interest was declared by the authors.

Funding: Not applicable.

Acknowledgements: 
This study was supported by Science and Technology Project of State Grid Corporation of China: Research and Application of Key Technology of Substation Joint Inspection based on Edge Computing and Incremental Learning (project number: 52051020000B).

\section{References}

[1] Long D. (2017) Application of Mobile Intelligent Inspection System in Substation Equipment Management. Energy and Power Engineering, 09(4):408-413.

[2] Lu S, Ying Z, Su J. (2017) Mobile robot for power substation inspection: a survey. IEEE/CAA Journal of Automatica Sinica, PP(99):1-18.

[3] Zhao X, Liu Z, Liu Y, Zhang B, Sui J, Jiang K. (2017) Structure Design and Application of Combination Track Intelligent Inspection Robot Used in Substation Indoor. Procedia Computer Science, 107:190-195.

[4] Xu HS, Chen TY, Zhang QP, Lu JX, Yang ZH. (2020) A Deep Learning and Depth Image based Obstacle Detection and Distance Measurement Method for Substation Patrol Robot. IOP Conference Series: Earth and Environmental Science, 582(1):012002 (9pp).

[5] Jiang KQ, Sun ZZ, Liu YC, Sui JC, Fu CG, Li YC. (2017) Development and Application of the Railtype Inspection Robot used in Substation Rooms. MATEC Web of Conferences, 139(5):00210.

[6] Zhao ZB, Xu GZ, Qi YC, Pan DF. (2016) An Intelligent On-Line Inspection and Warning System Based on Infrared Image for Transformer Bushings. Recent Advances in Electrical \& Electronic Engineering (Formerly Recent Patents on Electrical \& Electronic Engineering), 9(1):53-62.

[7] Hu Z, Wang Y, Li X, Liu D. (2015) Research and Utilization on RFID Label in Substation Inspection. Lecture Notes of the Institute for Computer Sciences Social Informatics \& Telecommunications Engineering, 142:232-239.

[8] Yang G, Xu W, Li J, Fu C, Zhou H, Zhang C, Shao G. (2017) Autonomous navigation method for substation inspection robot based on travelling deviation. IOP Conference Series Earth and Environmental Science, 69(1):012201.

[9] Luan Y Q, Yang W, Xiao P, Ma ZF, Wang HP. (2015) Design of Automatic Thermostat Control System for Substation Inspection Robot. Applied Mechanics \& Materials, 716-717(1):1457-1460.

[10] Zhao Z, Xu G, Qi Y, Pan D. (2016) An Intelligent On-Line Inspection and Warning System Based on Infrared Image for Transformer Bushings. Recent Advances in Electrical \& Electronic Engineering, 9(1).

[11] Zeng Q, Adu J, Liu J, Yang J, Xu Y, Gong M. (2019) Real-time adaptive visible and infrared image registration based on morphological gradient and C_SIFT. Journal of Real-Time Image Processing, 2019:1-13. 
[12] Gao Y, Zhao Y, Geng Y. (2017) Super-Resolution Restoration Method for Infrared Image of Power Line. Journal of Jilin University, 35(1):8-13.

[13] Chu X, Wang W, Yoon S C, Ni XZ, Heitschmidt GW. (2017) Detection of aflatoxin $B_{1}\left(A_{F B}\right)$ in individual maize kernels using short wave infrared (SWIR) hyperspectral imaging. Biosystems Engineering, 157:13-23.

[14] Jian L, Tan T. (2015) LS-SVM based substation circuit breakers maintenance scheduling optimization . International Journal of Electrical Power \& Energy Systems, 64:1251-1258.

[15] Gao J, Xu C, Zhang L, Liu S, Feng W, Xiong S, Tan S. (2017) Infrared Image Change Detection of Substation Equipment in Power System Using Markov Random Field. 2017 International Conference on Computing Intelligence and Information System (CIIS). IEEE Computer Society, 2017. 
Figures

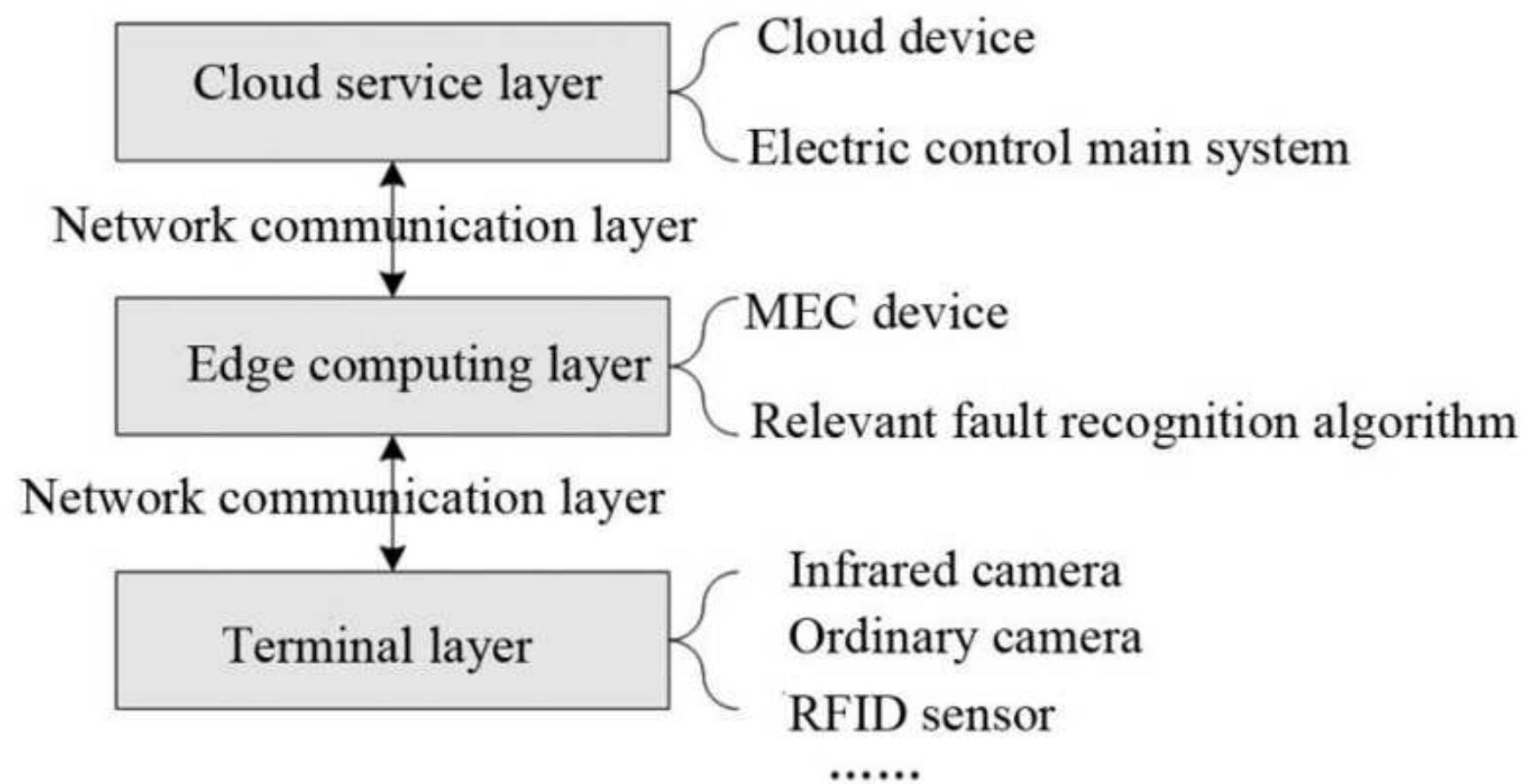

Figure 1

The system framework of the substation inspection robot based on edge computing 


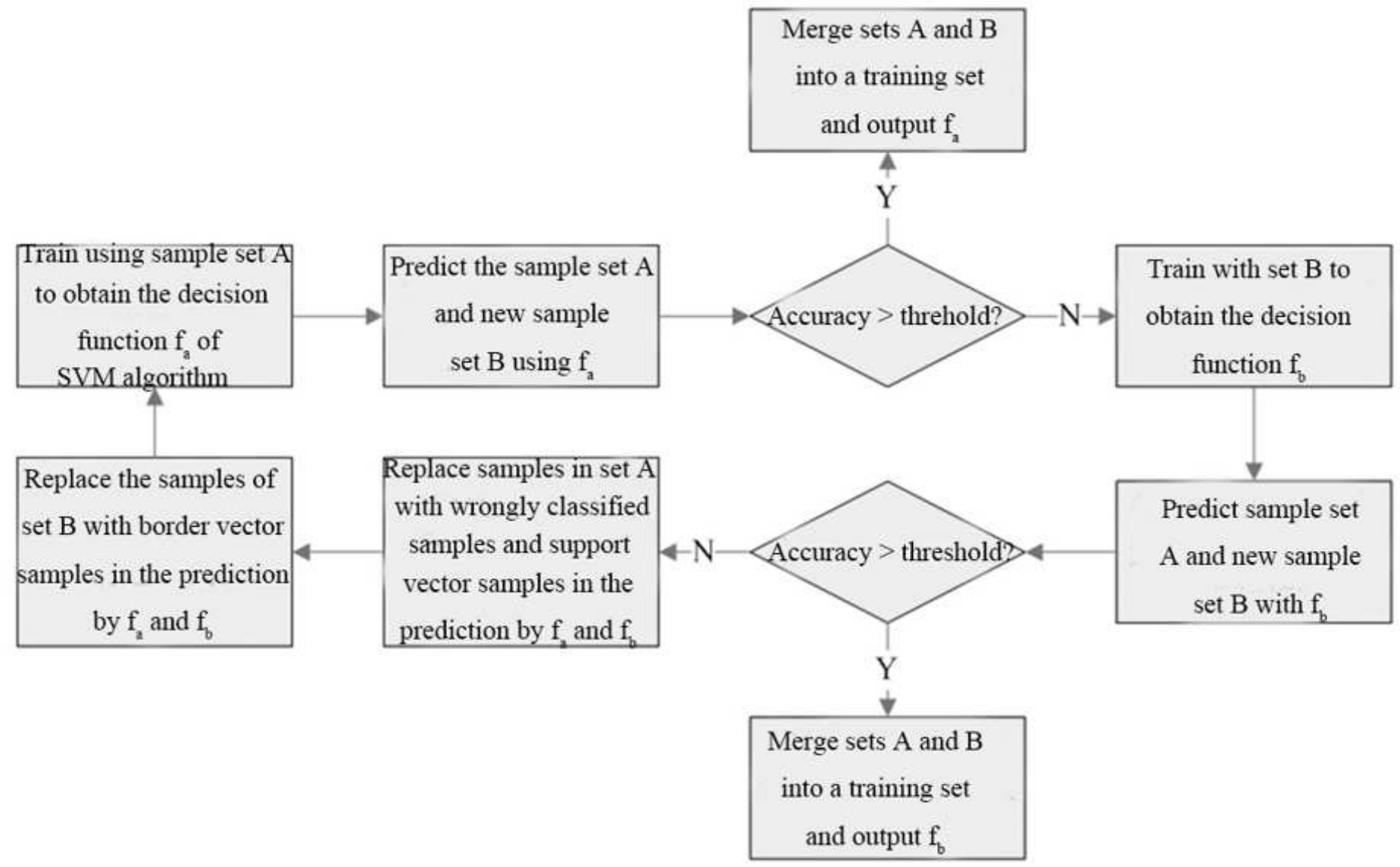

Figure 2

The training process of the improved SVM algorithm

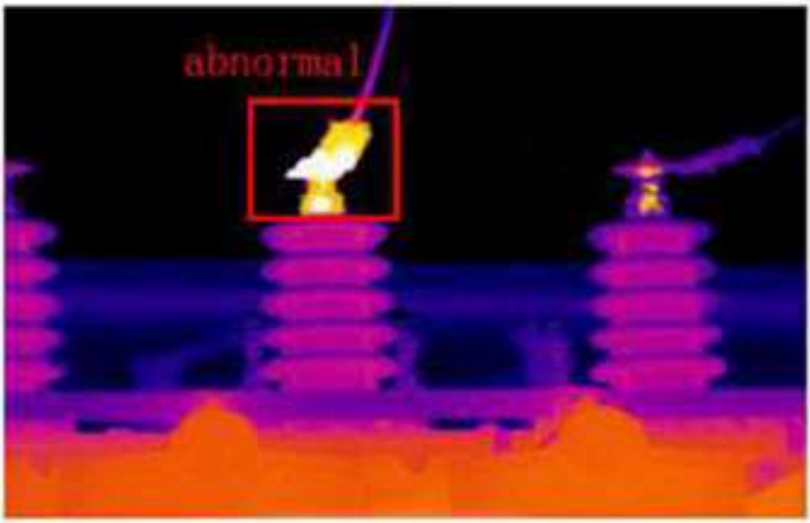

Traditional SVM algorithm

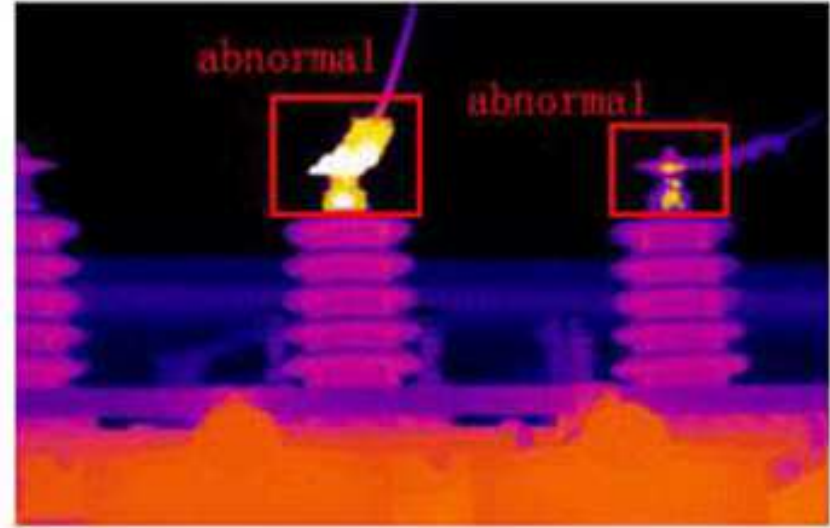

Improved SVM algorithm

\section{Figure 3}

Recognition results of two SVM algorithms for infrared images after incremental learning 


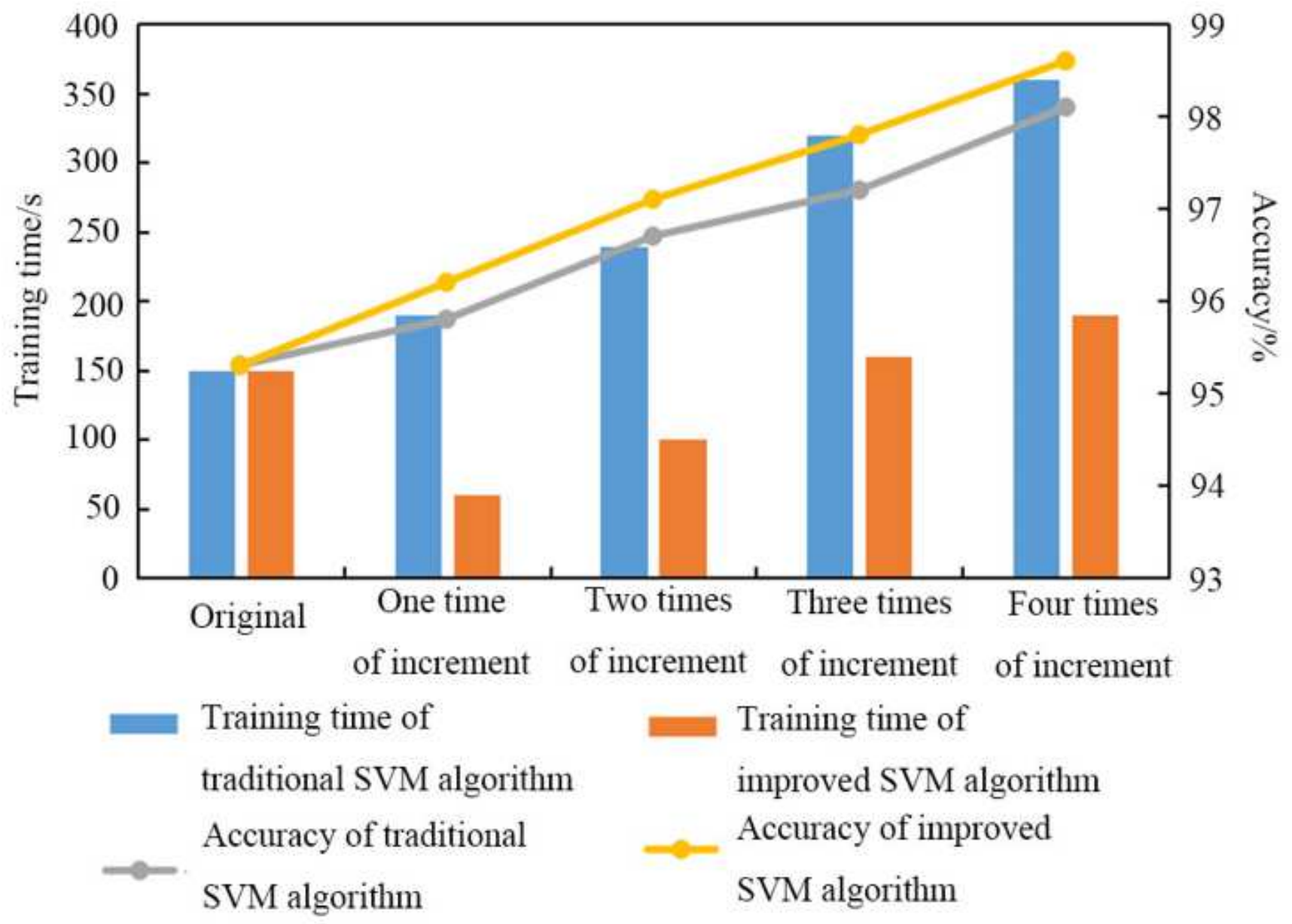

Figure 4

Results of the simulation experiment 